\title{
The high-resolution G-banded karyotype of Sus scrofa domestica L
}

\author{
O Galman, M Yerle, G Echard \\ Institut National de la Recherche Agronomique, \\ Laboratoire de Génétique Cellulaire, BP 27, 31326 Castanet-Tolosan, France
}

(Proceedings of the 9th European Colloquium on Cytogenetics of Domestic Animals; Toulouse-Auzeville, 10-13 July 1990)

high resolution banding / karyotype / pig / GTG-banding

The need for a high-resolution G-banded karyotype of the pig has been demonstrated following the publication by the Committee for a Standardized Karyotype of Sus scrofa (CSKSS, 1988) of its standard karyotype which was based on moderately extended G- and R-banded chromosomes. Rønne et al (1987) had presented a high-resolution $\mathrm{R}$-banded karyotype at the 541 band level but no corresponding G-banded karyotype has been published to date. To fill this gap, this paper describes a high-resolution GTG-banded pig karyotype at the 539 band level.

To obtain mitotic spreads at late prophase, early and mid-metaphase stages, pig lymphocytes were synchronized with methotrexate $\left(10^{-7} \mathrm{M}\right)$ for $18 \mathrm{~h}$ to block cells at $\mathrm{S}$ phase, then subsequently released by leucovorin $\left(3 \times 10^{-4} \mathrm{M}\right)$ and thymidine $\left(10^{-5} \mathrm{M}\right)$. Ethidium bromide $\left(2.5 \times 10^{-5} \mathrm{M}\right)$ and colcemid $\left(5 \times 10^{-7} \mathrm{M}\right)$ were employed 2 and $0.5 \mathrm{~h}$, respectively, before harvest. Hypotonic treatment, GTGbanding, photography and idiogram construction have been described elsewhere (Yerle et al, 1987).

The evolution of G-bands of each of the 38 pig chromosomes was analyzed from photographs of 52 well-spread and banded mitoses at progressive mitotic stages from metaphase (CSKSS standard) to late prophase. The final idiograms of the chromosomes with 4 haploid karyotypes at the 539 band level, shown in figure 1, take into consideration all 160 positive, 278 negative and 101 intermediate bands and subbands, according to their relative positions and staining intensities (Yerle et al, 1991). Using standard landmarks and nomenclature of major bands as reference points, the fate of each band was studied. For each chromosome, 3 or 4 intermediate stages were constructed to indicate which bands had subdivided, appeared or were retained in the final elongated stage. In most long chromosomes $(e g, 1,4,6,8$, $9,13,14$ and $\mathrm{X}$ ) landmarks established to recognize the chromosomes are no longer evident, for example band q.4.1 on chromosome 13. However, in other chromosomes some bands, eg, q.2.1.1 of chromosome 1, are still distinct in the definitive stage (fig 1). The persistence of centromeric dark bands in the telocentric chromosomes 

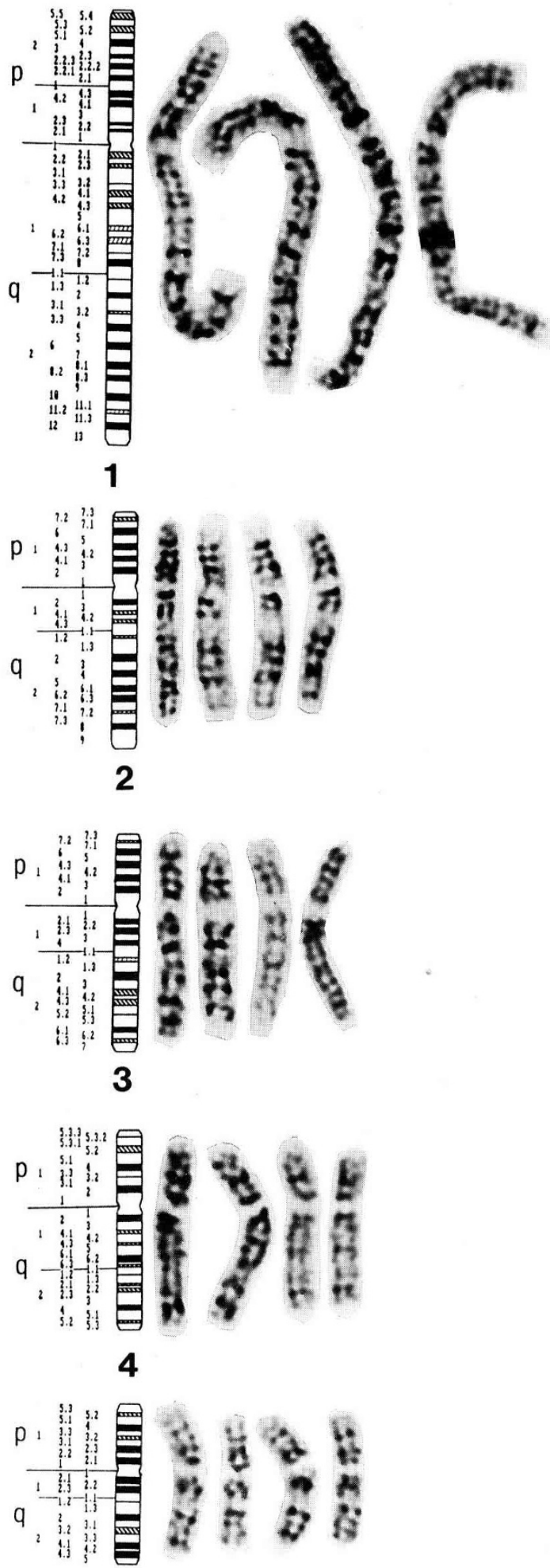

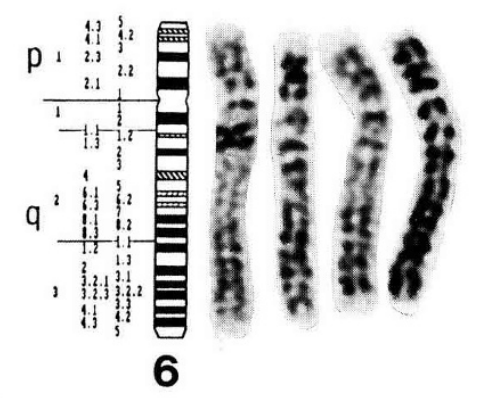

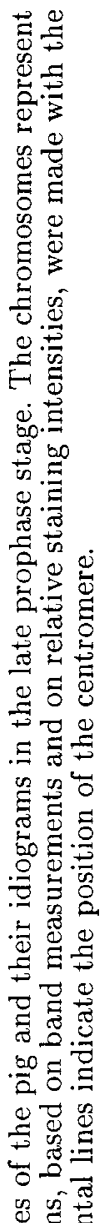
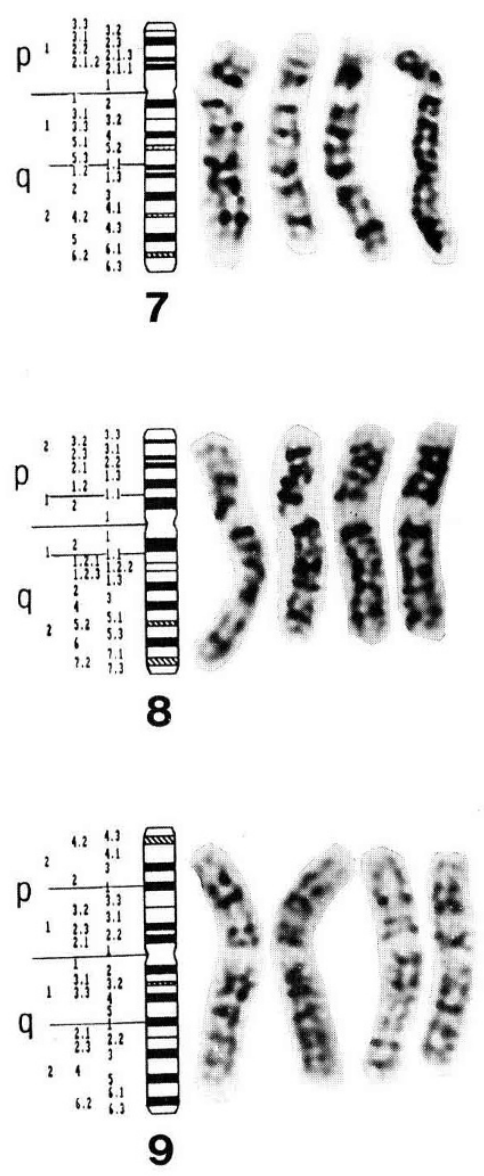

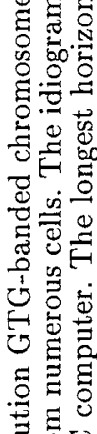

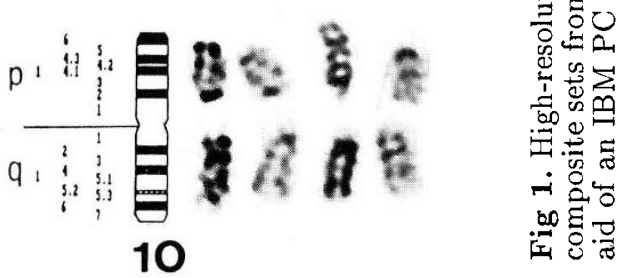



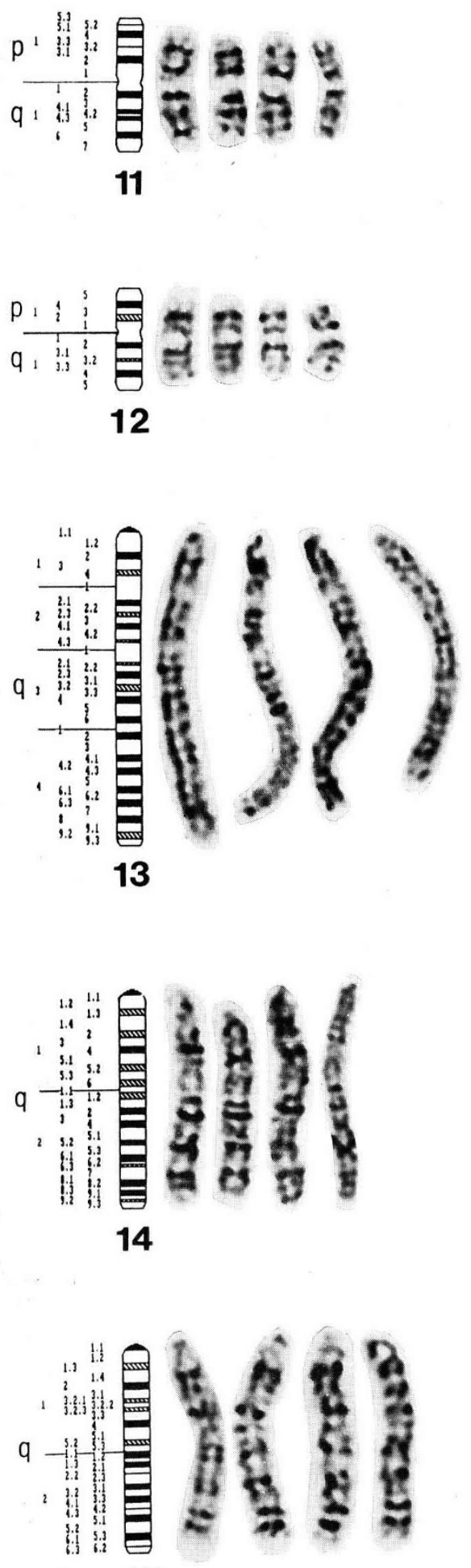
15
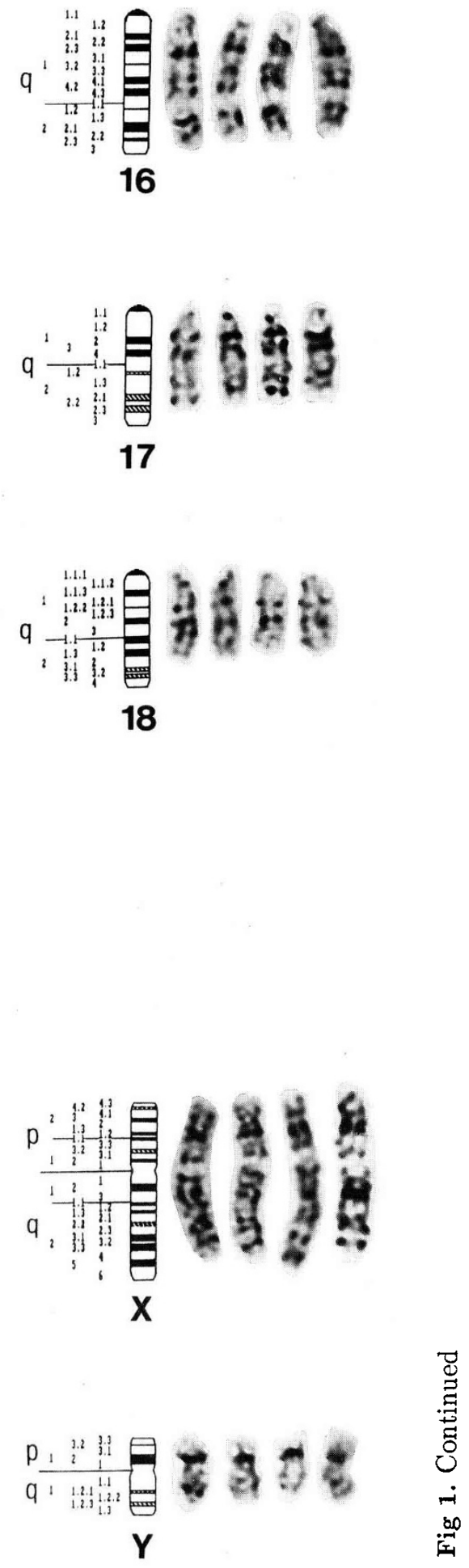
(13-18) from the standard to the final stage should also be noted. These bands, which correspond to (or overlap) the terminal $\mathrm{R}+$ bands (Rønne et al, 1987), are likely to be the centromeric heterochromatic C-bands which were also stained during GTG-banding.

The karyotype proposed here can be a useful tool for the study of comparative chromosome organization and the precise mapping of the porcine genome (Yerle $e t$ al, 1990).

\section{REFERENCES}

Committee for the Standardized Karyotype of Sus scrofa. (1988) Standard karyotype of the domestic pig. Hereditas 109, 151-157

Rønne M, Poulsen BS, Shibasaki Y, Flou S, Elberg JJ (1990) The high-resolution Rbanded karyotype of the domestic pig Sus scrofa domestica L. Cytobios 49, 103-109

Yerle M, Echard G, Gillois M (1987) The high resolution GTG-banding pattern of rabbit chromosomes. Cytogenet Cell Genet 45, 5-9

Yerle M, Gellin J, Dalens M, Galman O (1990) Localization on pig chromosome 6 of markers: GPI, APOE, ENO1 carried by chromosomes 1 and 19, using in situ hybridization. Cytogenet Cell Genet 54, 86-91

Yerle M, Galman O, Echard G (1991) The high resolution G-T-G banding pattern of pig chromosomes. Cytogenet Cell Genet 56, 45-47 